\title{
Alteración del gen AMELX en amelogénesis imperfecta. Una breve revisión
}

Omar Tremillo-Maldonado, ${ }^{1}$ Nelly Molina-Frechero, ${ }^{1}$ Rogelio González-González ${ }^{2}$ y Ronell Bologna-Molina ${ }^{3}$ 'Universidad Autónoma Metropolitana Unidad Xochimilco, Ciudad de México, México; '2Universidad Juárez del Estado de Durango, Facultad de Odontología, Departamento de Investigación, Durango, México; ${ }^{3}$ Universidad de la República, Escuela de Odontología, Área de Patología Molecular, Montevideo, Uruguay

\begin{abstract}
Resumen
La amelogénesis imperfecta es un grupo de trastornos de desarrollo del esmalte dental asociados principalmente con mutaciones en el gen AMELX. Clínicamente presenta diferentes fenotipos que afectan la estructura y función del esmalte, tanto de la dentición primaria como secundaria. El objetivo de este estudio fue realizar una revisión bibliográfica de las funciones y mutaciones de AMELX relacionadas con amelogénesis imperfecta. Se llevó a cabo una revisión bibliográfica en dos bases de datos: PubMed y Web of Science, usando las palabras clave "AMELX", "amelogenina", "amelogénesis imperfecta" y "mutación de AMELX". Fueron revisados 40 artículos y se encontró que AMELX es el gen predominante en el desarrollo del esmalte dental y de la amelogénesis imperfecta, alterando la estructura de la amelogenina. En los últimos años se han descrito las características en el proceso de amelogénesis imperfecta con diferentes fenotipos de esmalte hipoplásico o hipomineralizado y se han reportado diferentes mutaciones, con lo que se ha determinado la secuenciación del gen y las posiciones de las mutaciones.
\end{abstract}

PALABRAS CLAVE: AMELX. Amelogenina. Amelogénesis imperfecta. Mutación de AMELX.

\begin{abstract}
Amelogenesis imperfecta is a group of developmental disorders of the dental enamel that is mainly associated with mutations in the AMELX gene. Clinically, it presents different phenotypes that affect the structure and function of dental enamel both in primary and secondary dentition. The purpose of this study was to conduct a literature review on the AMELX functions and mutations that are related to amelogenesis imperfecta. A literature search was carried out in two databases: PubMed and Web of Science, using the keywords "AMELX", "amelogenin", "amelogenesis imperfecta" and "AMELX mutation". Forty articles were reviewed, with AMELX being found to be the predominant gene in the development of dental enamel and amelogenesis imperfecta by altering the structure of amelogenin. In the past few years, the characteristics of the amelogenesis imperfecta process have been described with different phenotypes of hypoplastic or hypo-mineralized enamel, and different mutations have been reported, by means of which the gene sequencing and the position of mutations have been determined.
\end{abstract}

KEY WORDS: AMELX. Amelogenin. Amelogenesis imperfecta. AMELX Mutation.

Correspondencia:

Omar Tremillo-Maldonado

E-mail: oatm88@msn.com
Fecha de recepción: 26-07-2017

Fecha de aceptación: 20-02-2018

DOI: 10.24875/GMM.18003604
Gac Med Mex. 2019;155:101-107

Disponible en PubMed www.gacetamedicademexico.com 


\section{Introducción}

El esmalte dental es un tejido altamente mineralizado; su formación, también conocida como amelogénesis, es un proceso que inicia cuando los ameloblastos células formadoras del esmalte depositan una delgada capa de matriz de esmalte rica en proteínas como amelogenina y, en menor proporción, enamelina ameloblastina y tuftelina, las cuales tienen la función de controlar el proceso de crecimiento de los cristales y la mineralización del esmalte; estas proteínas son degradadas por proteinasas secretadas por el ameloblasto. Este proceso finaliza con la mineralización de los cristales de esmalte, la eliminación de proteínas y la maduración, dando lugar a un tejido altamente mineralizado.

La amelogenina es la proteína predominante de la matriz de esmalte, por lo que su alteración se manifiesta clínicamente como amelogénesis imperfecta. ${ }^{1,2}$

La amelogénesis imperfecta es una enfermedad genética hereditaria que comprende un grupo de malformaciones del esmalte con diferentes trastornos del desarrollo que afectan la formación, mineralización y espesor del tejido, el cual puede presentar hipoplasia e hipomineralización, por lo que el diente puede adquirir un color amarillento, con textura áspera y pérdida de la translucidez del esmalte, así como de la función dental. ${ }^{1,3,4}$

Kim et al. ${ }^{5}$ manifiestan que la hipoplasia en el fenotipo del esmalte es causada por deficiencia en el desarrollo durante la secreción de matriz de esmalte y que las manifestaciones clínicas de hipomineralización pueden deberse a trastornos durante la maduración de la matriz. Existen diferentes manifestaciones clínicas de amelogénesis imperfecta, las cuales fueron descritas en 1988 por Witkop, quien clasificó las alteraciones de desarrollo del esmalte en cuatro grupos principales de acuerdo con la estructura del esmalte, con predominio o no de zonas hipoplásicas 0 hipomineralizadas. ${ }^{6}$

La prevalencia de amelogénesis imperfecta presenta una media mundial menor a $0.5 \%$, es decir, menos de un paciente entre 200.7 En distintas poblaciones, los reportes han mostrado diferencias de prevalencia de amelogénesis imperfecta; estudios epidemiológicos muestran rangos de 43:10 000 en Turquia, ${ }^{8}$ 14:10 000 en Suecia, ${ }^{9}$ 10:10 000 en Argentina, ${ }^{10} 1: 14000$ en Norteamerica ${ }^{4}$ y 1.25:10 000 en Israel. ${ }^{11}$ En estudios de anomalías dentales, la prevalencia de la amelogénesis imperfecta fue de tres en
1123 pacientes de la India, ${ }^{12} 0.08 \%$ en 1200 pacientes de Turquía, ${ }^{13}$ dos pacientes en 860 pacientes mexicanos $^{14}$ y cuatro en 478 pacientes en Brasil. ${ }^{15}$

Actualmente resulta importante la comprensión de la amelogénesis imperfecta, así como del papel de los genes y proteínas de formación de esmalte y sus variaciones genéticas. AMELX es uno de los principales genes que participan en la formación de la matriz de esmalte, así como en la señalización que requiere el ameloblasto para cumplir sus funciones durante el desarrollo del esmalte. Con base en esto, el propósito del presente trabajo fue realizar una revisión de la literatura científica con el objetivo de comprender el papel de AMELX en el proceso de desarrollo del esmalte dental, así como la importancia de sus alteraciones en el desarrollo de amelogénesis imperfecta.

\section{Método}

Se llevó a cabo un estudio descriptivo mediante una búsqueda de artículos de investigación y revisión publicados hasta 2016. La búsqueda se realizó en PubMed y Web of Science utilizando las palabras clave "AMELX", "amelogenina", "amelogénesis imperfecta" y "mutación de AMELX".

Se encontraron 74 artículos del año 1984 a 2016, todos en inglés, los cuales fueron sometidos a los siguientes criterios:

- Criterios de inclusión: estudios experimentales y clínicos de $A M E L X$ y de amelogenina, estudios de mutaciones en familias o poblacionales de amelogénesis imperfecta y revisiones bibliográficas sobre $A M E L X$, amelogenina y mutaciones.

- Criterios de exclusión: artículos que engloban otras proteínas derivadas del ameloblasto, como enamelina y ameloblastina, que se presentan en mínima proporción; artículos que estudian o detallan funciones de $A M E L X$ no relacionadas con amelogénesis imperfecta, estudios de amelogénesis imperfecta relacionados con otros genes, otras alteraciones y medicamentos.

En la Figura 1 se muestra cómo se realizó la exclusión de 34 artículos.

La búsqueda bibliográfica fue dividida en dos periodos: 1984-2008 y 2011-2016; se realizó una selección de las variaciones del gen $A M E L X$ consideradas patogénicas en el Centro Nacional para la Información Biotecnológica. ${ }^{16}$ 


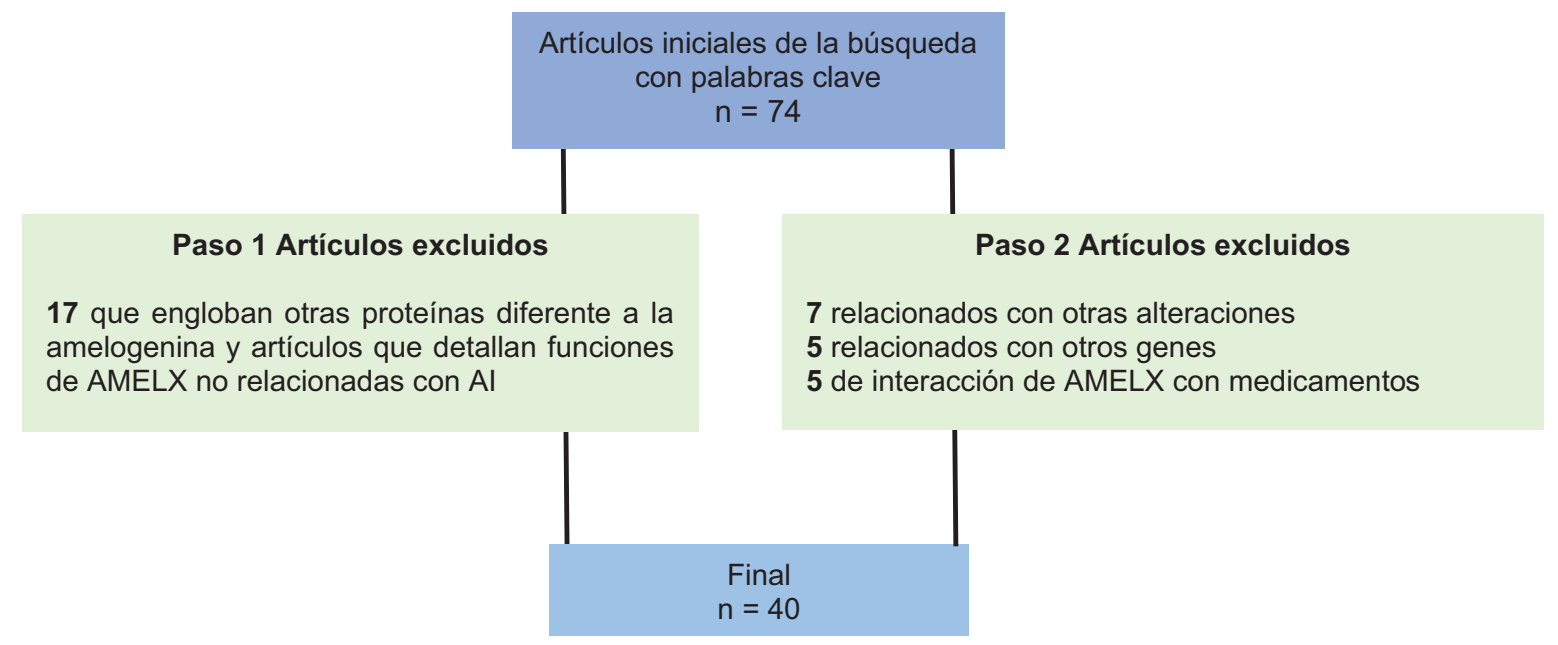

Figura 1. Pasos de exclusión y cómo se llegó al número final de artículos sobre amelogénesis imperfecta incluidos en la revisión. Al = amelogénesis imperfecta.

\section{Resultados}

Se encontraron artículos de 1984 a 2016, los cuales fueron organizados en los dos periodos indicados. Los trabajos del primer periodo (1984-2008) estuvieron enfocados a la secuenciación y funciones del gen y proteína en el proceso de amelogénesis; el primer estudio de mutación del gen data de 2007 (Tabla 1).

No se encontraron artículos con los criterios seleccionados en 2009 y 2010; a partir de 2011 se han realizado múltiples estudios del gen AMELX (Tabla 2). En las Tablas 1 y 2 se indican los descubrimientos más importantes relativos a $A M E L X$ y amelogenina a lo largo del tiempo; producto de esa revisión se obtuvo la información que se refiere a continuación.

\section{Estudio del gen AMELX}

AMEL es un gen que se encuentra en los cromosomas sexuales $X$ y $Y$. De acuerdo con el cromosoma al que pertenece se denomina AMELX y AMELY. AMEL del cromosoma sexual $X$ se encuentra en la región p22.3-p22.1 y está formado por más de 8000 pares de bases. ${ }^{17,18}$ AMEL se ha identificado en diferentes organismos desde los primeros anfibios. ${ }^{19}$

En 2008, Kawasaki relacionó a AMELX con la familia de genes que codifican para fosfoproteínas secretadas de unión al calcio (SCPP). La familia de genes SCPP incluye 21 genes que codifican para proteínas ácidas ricas en prolina y glutaminas que se encuentran en el cromosoma 4, con excepción de AMEL, que se ubica en los cromosomas sexuales $X$ y $Y^{20}$
Los genes SCPP están presentes en humanos, mamíferos y otras especies animales; esta familia de genes participa en la formación de tejidos calcificados, como hueso y dientes. ${ }^{21}$

En 2007, Iwase et al. reportaron la pertenencia de AMELX en el intrón 1 del gen ARHGAP6; mediante un estudio en diferentes mamíferos y anfibios encontraron que $A M E L X$ se encuentra dentro de dicho gen, lo que sugiere que esta localización se ha mantenido a lo largo de la historia desde los primeros anfibios. ${ }^{22}$ Prakash et al., mediante la inactivación del gen $A R H$ GAP6 en un estudio en ratones, reportaron que la supresión de dicho gen causa distintos fenotipos de esmalte y anomalías en otros tejidos o conductuales, por lo que sugieren que $A R H G A P$ no es fundamental en la formación del esmalte dental. ${ }^{23} \mathrm{Al}$ estudiar estos dos genes en una población de dos familias con amelogénesis imperfecta, Hu et al. reportaron en 2012 que $A M E L X$ es el principal gen que participa en amelogénesis imperfecta; demostraron que ARHGAP6 no tiene relación alguna con la formación del esmalte o tejido óseo. ${ }^{24}$

\section{Amelogenina}

AMELX en humanos codifica para la proteína amelogenina a través de siete exones, iniciando la traducción en el exón 2 y finalizando en el 7 , el cual solo codifica para un aminoácido., ${ }^{1,25}$

La amelogenina es una fosfoproteína secretada de unión a calcio, cuya secuencia es de 191 aminoácidos. ${ }^{26}$ Esta proteína puede ser codificada por AMELX 
Gaceta Médica de México. 2019;155

Tabla 1. Estudios del gen AMELX en el periodo 1984-2008

\begin{tabular}{|c|c|c|c|c|}
\hline Autor & Muestra & País & Año & Resultado principal \\
\hline Takagi et al. ${ }^{26}$ & Tejido bovino & Japón & 1984 & $\begin{array}{l}\text { - Realizó el primer estudio para secuenciar los de aminoácidos de } \\
\text { amelogenina bovina. }\end{array}$ \\
\hline Lau et al..$^{17}$ & Tejido humano y de ratón & $\begin{array}{l}\text { Estados } \\
\text { Unidos }\end{array}$ & 1989 & $\begin{array}{l}\text { - Primer estudio en humanos que ubicó AMEL en la región p22.1-p22.3 del } \\
\text { cromosoma } X \text { y en la región peri céntrica del cromosoma Y. } \\
\text { - En ratón solo en el cromosoma X. }\end{array}$ \\
\hline Salido et al. ${ }^{30}$ & Tejido humano & $\begin{array}{l}\text { Estados } \\
\text { Unidos }\end{array}$ & 1992 & - La amelogenina derivada de Y representa solo 10\% en la amelogénesis. \\
\hline $\begin{array}{l}\text { Sasaki y } \\
\text { Shimokawa }^{18}\end{array}$ & Tejido humano y animal & Japón & 1995 & $\begin{array}{l}\text { - Reportan la primera secuenciación de AMELX en } 1989 \text { por Shimokawa } \\
\text { et al. }\end{array}$ \\
\hline Iwase et al.22 & Pacientes & Japón & 2007 & - Reportan AMELX en el intrón 1 del gen Arhgap6. \\
\hline Richard et al..29 & Pacientes & Francia & 2007 & $\begin{array}{l}\text { - Reportan mutación de } A M E L X \text { con diferentes fenotipos hipoplásicos o } \\
\text { hipo mineralizados de esmalte. } \\
\text { - El exón } 6 \text { fue la región más variable. }\end{array}$ \\
\hline Kawasaki K. et al..$^{20}$ & $\begin{array}{l}\text { Revisión } \\
\text { bibliográfica }\end{array}$ & $\begin{array}{l}\text { Estados } \\
\text { Unidos }\end{array}$ & 2008 & $\begin{array}{l}\text { - Reportan pertenencia de AMELX a la familia de genes fosfoproteínas } \\
\text { secretadas de unión de calcio (SCPP). }\end{array}$ \\
\hline
\end{tabular}

Tabla 2. Estudios del gen AMELX en el periodo 2011-2016

\begin{tabular}{|c|c|c|c|c|}
\hline Autor & Muestra & País & Año & Resultado principal \\
\hline Lee et al. ${ }^{45}$ & Pacientes con Al & Corea & 2011 & $\begin{array}{l}\text { - Reporta nueva mutación del gen AMELX con fenotipo hipo mineralizado } \\
\text { incluso en dientes no erupcionados. } \\
\text { - AMELX con } 7 \text { exones la traducción comienza en el exón } 2 \text { y el exón } 7 \\
\text { codifica a un solo aminoácido. }\end{array}$ \\
\hline Hu et al. ${ }^{24}$ & Pacientes con Al & Estados Unidos & 2012 & $\begin{array}{l}\text { - La supresión del gen Arhgap6, excepto el intrón } 1 \text { no demostró cambios } \\
\text { en el esmalte. }\end{array}$ \\
\hline Fukuda et al..$^{27}$ & Tejido animal ratón & Japón & 2013 & - Reportan interacción AMELX con genes formadores de tejido óseo. \\
\hline Jacques et al. ${ }^{39}$ & Modelo animal ratones & Francia & 2014 & $\begin{array}{l}\text { - Participación de AMELX en matriz de esmalte y en crecimiento de } \\
\text { maxilares. }\end{array}$ \\
\hline Cho et al. ${ }^{3}$ & Pacientes con Al & Corea & 2014 & $\begin{array}{l}\text { - Reportan la producción de proteínas alternativas del gen AMELX, } \\
\text { aunque sus funciones no son claras. }\end{array}$ \\
\hline Guo et al. ${ }^{37}$ & Tejido animal ratón & Estados Unidos & 2015 & $\begin{array}{l}\text { - Reportan que la deficiencia de amelogenina favorece la presencia de } \\
\text { zonas ácidas en el esmalte en desarrollo afectando su maduración. }\end{array}$ \\
\hline Le et al. ${ }^{40}$ & Tejido animal ratón & Estados Unidos & 2016 & $\begin{array}{l}\text { - Reportan que el exón } 4 \text { de AMELX participa en la formación de } \\
\text { amelogenina además de participar en la formación de tejido óseo. }\end{array}$ \\
\hline
\end{tabular}

y $A M E L Y$, sin embargo, Salido et al. encontraron que AMELY representa solo el $10 \%$ durante la amelogénesis, por lo que $A M E L X$ es el principal gen formador de amelogenina en dicho proceso. ${ }^{27-30}$ En un estudio del cromosoma $Y$, Jobling et al. reportaron que AMELY tiene poca participación en la formación del esmalte y no tiene expresión en otros tejidos. ${ }^{31}$ Además, otros autores lograron establecer que la amelogenina formada por $A M E L Y$ no logra cubrir las deficiencias en el desarrollo de esmalte provocadas por alteraciones en $A M E L X$, por lo que consideran que se deben realizar más estudios para determinar el participación de la amelogenina generada por AMELY.32
La amelogenina es la proteína más abundante en el proceso de formación del esmalte, ya que comprende $90 \%$ de la matriz de esmalte. Existen diferentes variantes de esta proteína conocidas como isoformas, resultado del empalme alternativo durante el proceso de transcripción y traducción de la proteína; estas variaciones pueden ser desde diferentes longitudes hasta cambios significativos en la estructura de la proteína. Actualmente no se conoce del todo si existen diferencias funcionales en las diferentes isoformas de la proteína, por ejemplo, la amelogenina M180 constituye la isoforma más común, genera un esmalte más resistente al desgaste y más frágil a la fractura, en comparación 


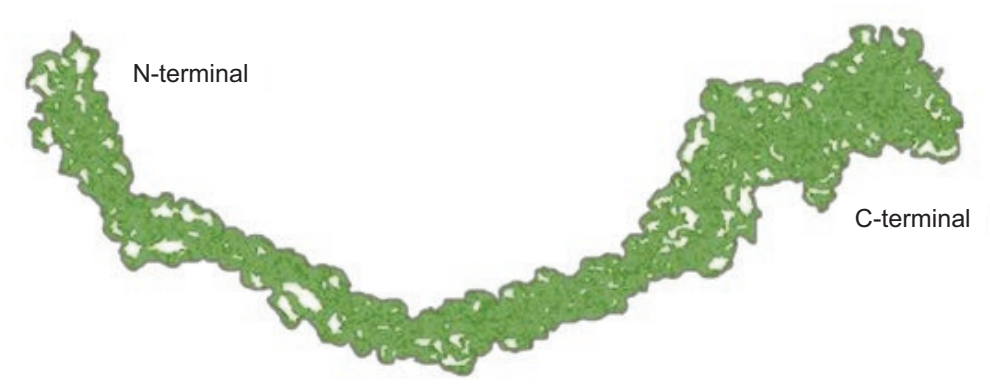

Figura 2. Simulación gráfica de amelogenina porcina. Modificado de Delak et al. ${ }^{35}$

con el esmalte normal. En estudios realizados en ratones fue posible identificar la modificación de la proteína $y$, por lo tanto, de los fenotipos de esmalte. ${ }^{33}$ Dichas variaciones han sido relacionadas con trastornos de la estructura del esmalte y amelogénesis imperfecta, pero, por otra parte, se ha sugerido que en otras especies animales la existencia de estas variantes de amelogenina se debe a las exigencias biomecánicas que se ejercen sobre el esmalte. ${ }^{34}$

En 2009, Delak et al., con base en la bioinformática, crearon un modelo simulado en tres dimensiones de la amelogenina porcina; encontraron que la estructura de amelogenina forma parte de un grupo de proteínas que tiene la capacidad de mantener una forma desplegada o contraída debido a que realiza diferentes funciones con cada forma. ${ }^{35}$ En la Figura 2 se representa una simulación gráfica de la amelogenina porcina desplegada y se señalan los grupos terminales.

La amelogenina cumple funciones como inducir la formación del cristal de apatita, controlando su forma y crecimiento; protege al cristal y al ameloblasto al controlar los cambios de pH; además, participa en la señalización con osteoblastos en el proceso de formación del tejido óseo.

El crecimiento de los cristales de esmalte se lleva a cabo con el proceso de mineralización; por medio de nanoesferas, la amelogenina guía el crecimiento de los cristales a lo largo, evitando que se unan entre sí. Cuando este proceso está desarrollado se produce la eliminación gradual de la matriz de esmalte, constituida principalmente por amelogenina, y los cristales comienzan a crecer en anchura, quedando unidos lateralmente., ${ }^{1,36}$

Guo et al. reportaron que la amelogenina desempeña un papel importante en el control del $\mathrm{pH}$, ya que cuando existe deficiencia de amelogenina se presentan zonas ácidas en el esmalte en desarrollo que afectan la maduración de este; al ocurrir una caída del $\mathrm{pH}$, la proteína se encuentra alterada en su estructura y función, afectando así el proceso de amelogénesis, por ello se afirma que la amelogenina posee el mecanismo de control de $\mathrm{pH} \cdot{ }^{37}$ En el estudio de Yan Q. et al. se destaca la importancia de que la amelogenina sea secretada en cantidades necesarias para que cumpla la función de proteger al ameloblasto de los cambios externos del pH. ${ }^{38}$

Además de lo anterior, Jacques et al., mediante técnicas de biología molecular e inmunohistoquímica, observaron que la amelogenina participa en la señalización a corta distancia con células formadoras de hueso, guiando así la morfología del hueso local mandibular. Estos autores plantean un doble papel de la amelogenina: como componente de matriz de esmalte y como moléculas de factor de crecimiento en el hueso que rodea al diente. ${ }^{39}$ Estas observaciones son confirmadas por Le et al. en un estudio realizado en células de ratón: relacionan la interacción del miRNA del exón 4 de $A M E L X$ directamente con la actividad celular de los osteoblastos. ${ }^{40}$

La degradación de las amelogeninas se realiza de forma continua desde que comienza la secreción, permitiendo que el cristal de apatita alcance su tamaño normal; el proceso de eliminación de proteínas termina hasta que el esmalte está totalmente mineralizado. ${ }^{41,42}$ En este proceso debe existir un equilibrio de $\mathrm{pH}$, de ahí que Lu et al. manifiesten que las proteinasas no eliminan la amelogenina cuando el $\mathrm{pH}$ disminuye, lo que impide la correcta degradación de amelogenina que resulta en la alteración de la estructura del esmalte.43

\section{Alteración del gen AMELX}

En un reporte de caso de 2010, Lindermeyer et al. relacionaron el genotipo y fenotipo de la amelogénesis imperfecta. Reportaron que la correcta eliminación de la 


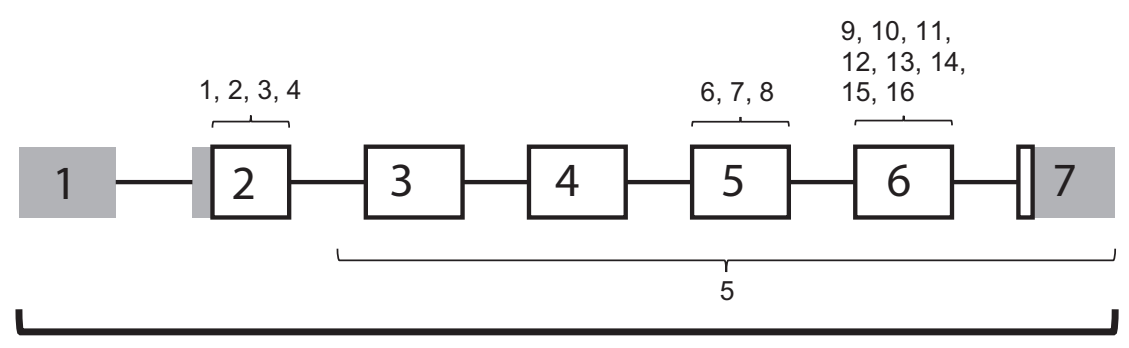

AMELX

Figura 3. Esquema de los siete exones de AMELX. En blanco se simbolizan los exones 2 al 7 que codifican para amelogenina. Se ubican las 16 mutaciones conocidas de AMELX para amelogénesis imperfecta. Modificado de Hu et al. ${ }^{24}$

amelogenina es fundamental para el desarrollo de un esmalte sano, por lo que es importante que esta proteína no se encuentre mutada 0 en cantidades incorrectas. ${ }^{44}$

En la actualidad, los estudios de amelogénesis imperfecta se han enfocado en la identificación de mutaciones que alteran la síntesis de la proteína amelogenina, la cual al presentar diferentes características no es reconocida por las proteinasas encargadas de su eliminación. Por esa falta de identificación, la amelogenina permanece en exceso, que se traduce en un esmalte hipomineralizado al finalizar su formación. ${ }^{29}$

Debido a la importancia de AMELX en el desarrollo del esmalte dental, las mutaciones de este gen provocan variaciones en la proteína, afectando el desarrollo del esmalte. Actualmente se conocen 16 mutaciones de $A M E L X$ que modifican a amelogenina y causan amelogénesis imperfecta con diferentes características de hipomineralización e hipoplasia de esmalte no relacionadas con otra enfermedad. La afectación en el desarrollo de los cristales y su organización, así como las funciones de señalización y protección de la amelogeninas pueden causar distintos fenotipos de amelogénesis imperfecta, con diferencias en la severidad según la localización de las mutaciones en $A M E L X$. ${ }^{25,36,45,46}$

Hu et al. realizaron una revisión de las 16 mutaciones descritas en la literatura y presentaron una representación gráfica del gen $A M E L X$, los siete exones que lo componen, así como las zonas de los exones que codifican para la proteína; además, ubicaron lo exones donde se presenta cada mutación (Figura 3). ${ }^{25}$

Los estudios de mutaciones del gen AMELX resultan importantes ya que existen variaciones genotípicas en las poblaciones; la identificación de las mutaciones en distintos grupos humanos repercutirá en la comprensión de $A M E L X$ y la amelogénesis imperfecta.

Wright et al. estudiaron 463 sujetos de 54 familias con alguna variedad de amelogénesis imperfecta, en quienes realizaron la secuenciación de distintos genes; encontraron mutaciones en el gen $A M E L X$, que pueden ser ligadas a distintos grados visibles de hipomineralización e hipoplasia del esmalte, desde pequeñas fosas en el esmalte y ranuras, hasta adelgazamiento o falta del tejido. Reportaron tres diferentes mutaciones de AMELX asociadas con amelogénesis imperfecta en dicha población. ${ }^{46}$

Otras investigaciones encontraron las mutaciones ya reportadas en $40 \%$ de familias y $60 \%$ de individuos con amelogénesis imperfecta; en el resto no fue posible identificarlas, lo que sugiere que aún existen mutaciones sin estudiar o regiones no codificantes de los genes que participan en el desarrollo de este padecimiento, además de la posibilidad de nuevos genes no incluidos en la etiología. ${ }^{46,47}$

Las mutaciones en AMELX contribuyen a la alteración de la estructura de la amelogenina, lo que puede alterar la formación de esmalte mediante distintas vías, como la alteración de las funciones de la proteína, además de su interacción con el ameloblasto y otras proteínas importantes en el desarrollo de dicho tejido. Las principales variaciones de $A M E L X$ son c. $152 \mathrm{C}>\mathrm{T}, \mathrm{c} .571 \mathrm{G}>\mathrm{T}, \mathrm{c} .208 \mathrm{C}>\mathrm{A}, \mathrm{c} .2 \mathrm{~T}>\mathrm{C}, \mathrm{c} .11 \mathrm{G}>\mathrm{C}$ de un solo nucleótido y c.155delC, c.14_22delTTTTATTTG, c.473delC y c.541delC, mutaciones por deleción de distintos nucleótidos, las cuales se traducen en amelogénesis imperfecta con hipoplasia e hipomineralización dominante ligada al cromosoma $X^{16,48,49}$

Producto de esta revisión podemos concluir que $A M E L X$ es el gen con mayor predominio en el desarrollo del esmalte y de la amelogénesis imperfecta. La amelogenina induce la formación del cristal de apatita y controla el pH y la señalización con células formadoras del tejido óseo que rodea al diente; las mutaciones de $A M E L X$ produce alteración en las funciones de la esta proteína, como la falta de interacción célula y matriz de esmalte, cambios de $\mathrm{pH}$, crecimiento anormal de los cristales de esmalte $y$, posteriormente, la interacción de la amelogenina con 
las proteinasas. Además, en la secuencia del gen se ha identificado que la posición de las mutaciones genera distintos fenotipos de amelogénesis imperfecta, con manifestación clínica de esmalte hipomineralizado o hipoplásico.

\section{Agradecimientos}

A CONACyT por el apoyo No. 284071, al Doctorado en Ciencias Biológicas y de la Salud y al Departamento de Atención a la Salud de la Universidad Autónoma Metropolitana Xochimilco.

\section{Bibliografía}

1. Hu JC, Chun YH, Al Hazzazzi T, Simmer JP. Enamel formation and amelogenesis imperfecta. Cells Tissues Organs. 2007;186:78-85.

2. Prasad MK, Laouina S, El-Alloussi M, Dollfus H, Bloch-Zupan A. Amelogenesis imperfecta: 1 family, 2 phenotypes, and 2 mutated genes. J Dent Res. 2016;95:1457-1463.

3. Cho ES, Kim KJ, Lee KE, Lee EJ, Yun CY, Lee MJ, et al. Alteration of conserved alternative splicing in AMELX causes enamel defects. J Dent Res. 2014;93:980-987.

4. Crawford PJ, Aldred M, Bloch-Zupan A. Amelogenesis imperfecta. Orphanet J Rare Dis. 2007;2:17.

5. Kim JW, Simmer JP, Hart TC, Hart PS, Ramaswami MD, Bartlett JD. MMP-20 mutation in autosomal recessive pigmented hypomaturation amelogenesis imperfecta. J Med Genet. 2005;42:271-275.

6. Witkop CJ. Amelogenesis imperfecta, dentinogenesis imperfecta and dentin dysplasia revisited: problems in classification. J Oral Pathol. 1988;17:547-553

7. Gadhia K, McDonald S, Arkutu N, Malik K. Amelogenesis imperfecta: an introduction. Br Dent J. 2012;212:377-379.

8. Altug-Atac AT, Erdem D. Prevalence and distribution of dental anomalies in orthodontic patients. Am J Orthod Dentofacial Orthop. 2007;131:510-514

9. Bäckman B, Holm A K. Amelogenesis imperfecta: prevalence and incidence in a northern Swedish county. Community Dent Oral Epidemiol. 1986;14:43-47.

10. Sedano HO. Congenital oral anomalies in Argentinian children. Community Dent Oral Epidemiol. 1975;3:61-63.

11. Chosack A, Eidelman E, Wisotski I, Cohen T. Amelogenesis imperfecta among Israeli Jews and the description of a new type of local hypoplastic autosomal recessive amelogenesis imperfecta. Oral Surg Oral Med Oral Pathol. 1979;47:148-156.

12. Gupta SK, Saxena P, Jain S, Jain D. Prevalence and distribution of selected developmental dental anomalies in an Indian population. J Oral Sci. 2011;53:231-238.

13. Bilge NH, Yeşiltepe S, Törenek-Ağırman K, Çağlayan F, Bilge OM. Investigation of prevalence of dental anomalies by using digital panoramic radiographs. Folia Morphol (Warsz). 2018;77:323-328.

14. Herrera-Atoche JR, Agüayo-De-Pau MD, Escoffié-Ramírez M Aguilar-Ayala FJ, Carrillo-Ávila BA, Rejón-Peraza ME. Impacted maxillary canine prevalence and its association with other dental anomalies in a Mexican population. Int J Dent. 2017;2017:7326061.

15. Goncalves-Filho AJ, Moda LB, Oliveira RP, Ribeiro AL, Pinheiro JJ, Alver-Junior SR. Prevalence of dental anomalies on panoramic radiographs in a population of the state of Pará, Brazil. Indian J Dent Res. 2014; 25:648-652.

16. Database of Single Nucleotide Polymorphisms (dbSNP). EE. UU.: National Center for Biotechnology Information/National Library of Medicine; 2016.

17. Lau EC, Mohandas TK, Shapiro LJ, Slavkin HC, Snead ML. Human and mouse amelogenin gene loci are on the sex chromosomes. Genomics. 1989;4:162-168.

18. Sasaki S, Shimokawa H. The amelogenina gene. Int J Dev Biol. 1995;39:127-133.

19. Sire JY, Delgado S, Fromentin D, Girondot M. Amelogenin: lessons from evolution. Arch Oral Biol. 2005;50:205-212.

20. Kawasaki K, Weiss KM. SCPP gene evolution and the dental mineralization continuum. J Dent Res. 2008;87:520-531.

21. Kawasaki K. The SCPP gene repertoire in bony vertebrates and graded differences in mineralized tissues. Dev Genes Evol. 2009;219: 147-157.
22. Iwase M, Kaneko S, Kim H, Satta Y, Takahata N. Evolutionary history of sex-linked mammalian amelogenin genes. Cells Tissues Organs. 2007;186:49-59.

23. Prakash SK, Gibson CW, Wright JT, Boyd C, Cormier T, Sierra R, et al. Tooth enamel defects in mice with a deletion at the Arhgap6/AmelX locus. Calcif Tissue Int. 2005;77:23-29.

24. Hu JC, Chan HC, Simmer SG, Seymen F, Richardson AS, Hu Y, et al. Amelogenesis imperfecta in two families with defined AMELX deletions in ARHGAP6. PLoS One. 2012;7:e52052.

25. Cho ES, Kim KJ, Lee KE, Lee EJ, Yun CY, Lee MJ, et al. Alteration of conserved alternative splicing in AMELX causes enamel defects. J Dent Res. 2014;93:980-987.

26. Takagi T, Suzuki M, Baba T, Minegishi K, Sasaki S. Complete amino acid sequence of amelogenina in developing bovine enamel. Biochem Biophys Res Commun. 1984;121:592-597.

27. Fukuda T, Sanui T, Toyoda K, Tanaka U, Taketomi T, Uchiumi T, et al. Identification of novel amelogenin-binding proteins by proteomics analysis. PLoS One. 2013;8:e78129.

28. Kim YJ, Kim YJ, Kang J, Shin TJ, Hyun HK, Lee SH, et al. A novel AMELX mutation causes hypoplastic amelogenesis imperfecta. Arch Oral Biol. 2017;76:61-65.

29. Richard B, Delgado S, Gorry P, Sire JY. A study of polymorphism in human AMELX. Arch Oral Biol. 2007;52:1026-1031.

30. Salido EC, Yen PH, Koprivnikar K. Yu LC, Shapiro LJ. The human enamel protein gene amelogenin is expressed from both the $X$ and the Y chromosomes. Am J Hum Genet. 1992;50:303-316.

31. Jobling MA, Lo IC, Turner DJ, Bowden GR, Lee AC, Xue Y, et al. Structural variation on the short arm of the human $Y$ chromosome: recurrent multigene deletions encompassing amelogenin Y. Hum Mol Genet. 2007;16:307-316.

32. Bartlett JD. Dental enamel development: proteinases and their enamel matrix substrates. ISRN Dent. 2013;684607.

33. Pugach MK, Suggs C, Li Y, Wright JT, Kulkarni AB, Bartlett JD, et al. M180 amelogenin processed by MMP20 is sufficient for decussating murine enamel. J Dent Res. 2013;92:1118-1122.

34. Rauth RJ, Potter KS, Ngan AY, Saad DM, Mehr R, Luong VQ, et al. Dental enamel: genes define biomechanics. J Calif Dent Assoc. 2009;37:863-868.

35. Delak K, Harcup C, Lakshminarayanan R, Sun Z, Fan Y, Moradian-Oldak J, et al. The tooth enamel protein, porcine amelogenin, is an intrinsically disordered protein with an extended molecular configuration in the monomeric form. Biochemistry. 2007;48:2272-2281.

36. Seymen F, Park JC, Lee KE, Lee HK, Lee DS, Koruyucu M, et al. Novel MMP20 and KLK4 mutations in amelogenesis imperfecta. J Dent Res. 2015:94:1063-1069.

37. Guo J, Lyaruu DM, Takano Y, Gibson CW, DenBesten PK, Bronckers AL. Amelogenins as potential buffers during secretory-stage amelogenesis. J Dent Res. 2015;94:412-420.

38. Yan Q, Zhang Y, Li W, DenBesten PK. Micromolar fluoride alters ameloblast lineage cells in vitro. J Dent Res. 2007;86:336-340.

39. Jacques J, Hotton D, De-La-Dure-Molla M, Petit S, Asselin A, Kulkarni AB, et al. Tracking endogenous amelogenin and ameloblastin in vivo. PLoS One. 2014;9:e99626.

40. Le MH, Warotayanont R, Stahl J, Den-Besten PK, Nakano Y. Amelogenin EXon4 forms a novel miRNA that directs ameloblast and osteoblast differentiation. J Dent Res. 2016;95:423-429.

41. Fanjul-Fernández M, Folgueras AR, Cabrera S, López-Otín C. Matrix metalloproteinases: evolution, gene regulationand functional analysis in mouse models. Biochim Biophys Acta.2010;1803:3-19.

42. Kawasaki K, Suzuki T. Molecular evolution of matrix metalloproteinase 20. Eur J Oral Sci. 2011;119:247-253.

43. Lu Y, Papagerakis P, Yamakoshi Y, Hu JC, Bartlett JD, Simmer JP. Functions of KLK4 and MMP-20 in dental enamel formation. Biol Chem. 2008;389:695-700.

44. Lindemeyer RG, Gibson CW, Wright TJ. Amelogenesis imperfecta due to a mutation of the enamelin gene: clinical case with genotype-phenotype correlations. Pediatr Dent. 2010;32:56-60.

45. Lee KE, Lee SK, Jung SE, Song SJ, Cho SH, Lee ZH, et al. A novel mutation in the AMELX gene and multiple crown resorptions. Eur J Oral Sci. 2011;119:324-328.

46. Wright JT, Torain M, Long K, Seow K, Crawford P, Aldred MJ, et al. Amelogenesis imperfecta: genotype-phenotype studies in 71 families. Cells Tissues Organs. 2011;194:279-283.

47. Kim JW, Simmer JP, Lin BP, Seymen F, Bartlett JD, Hu JC. Mutational analysis of candidate genes in 24 amelogenesis imperfecta families. Eur J Oral Sci. 2006;114:3-12.

48. Bartlett JD, Simmer JP. Kallikrein-related peptidase-4 (KLK4): role in enamel formation and revelations from ablated mice. Front Physiol. 2014;5:240.

49. Barron MJ, Brookes SJ, Kirkham J, Shore RC, Hunt C, Mironov A, et al. A mutation in the mouse Amelx tri-tyrosyl domain results in impaired secretion of amelogenin and phenocopies human X-linked amelogenesis imperfecta. Hum Mol Genet. 2010;19:1230-1247. 